\title{
Thermal diffusivity of ferrofluids as a function of particle size determined using the mode-mismatched dual-beam thermal lens technique
}

\author{
V. M. Lenart and N. G. C. Astrath \\ Department of Physics, State University of Maringá, Maringá, PR, Brazil. \\ R. F. Turchiello \\ Physics Department, Federal University of Technology, Ponta Grossa, PR, Brazil. \\ G. F. Goya \\ Department of Condensed Matter Physics, Aragon Institute of Nanoscience, Zaragoza, Spain. \\ S. L. Gómez \\ Physics Department, State University of Ponta Grossa, Ponta Grossa, PR, Brazil.*
}

(Dated: February 7, 2018)

\begin{abstract}
Ferrofluids are colloids of superparamagnetic nanoparticles that are envisaged for use in hyperthermia, which is based on nonradiative relaxation after interaction with a high-frequency magnetic field or light. For such applications, an important parameter is the thermal diffusivity. In this communication, we present an experimental study of the dependence of thermal diffusivity of ferrofluids on the size of the magnetite nanoparticles by employing the mode-mismatched thermal lens technique. The results show a huge enhancement of the thermal diffusivity by increasing the average size of the nanoparticles, while the number density of the nanoparticles is maintained as constant.
\end{abstract}

Keywords: ferrofluid, thermal lens, thermal diffusivity.

\section{INTRODUCTION}

Ferrofluids or magnetic colloids are a kind of nanofluid, a stable colloidal suspension of nanoparticles (NPs), obtained by dispersing superparamagnetic NPs in a carrying fluid [1]. Such colloids have found numerous applications in many fields, for example inertia-damping apparatuses, vacuum seals [2], biomedical applications, such as drug delivery and imaging [3], high-frequency magnetic field hyperthermia [4,5], and optically activated hyperthermia [6]. For the last exemples, an important parameter is the thermal diffusivity $(D)$, which measures the rate of heat transfer following absorption of electromagnetic radiation and is defined as $D=\kappa / \rho c_{p}$, where $\kappa$ is the thermal conductivity, $\rho$ is the mass density, and $c_{p}$ is the specific heat capacity. The heat capacity and thermal conductivity of nanocolloids are known to depend on many factors, such as the size and concentration of the NPs and the aggregation state. The dependence on the size of the NPs arises mainly from the higher surface-tovolume ratio of nano-sized particles. It is known that the heat capacity of nanocrystals is higher than that of the bulk material [7-9]. On the other hand, the specific heat capacity of solids is lower than that of fluids, so the addition of nanocrystals to a fluid leads to a nanofluid with a lower specific heat capacity than that of the undoped liquid, as predicted by the thermal equilibrium model [10], although opposite behavior has also been observed [11]. Usually highly concentrated ferrofluids exhibit a strong

*sgomez@uepg.br dependence of $c_{p}$ on concentration, decreasing the value of the specific heat capacity by increasing the concentration $[12,13]$.

The thermal conductivity of nanofluids has been studied using many techniques [14]. It is known that the addition of a small quantity of nanocrystals of a material of high thermal conductivity to a fluid leads to a colloid of enhanced thermal conductivity, depending on NP size and concentration, among other factors [15]. It has been shown that the thermal conductivity increases as a function of the concentration of NPs and, at constant concentration of the nanoparticulate phase, decreases by increasing the size of the NPs [16]. Another contribution to the thermal conductivity of a nanofluid is the capping of the NPs [17]. As shown by Lenin et al., the thermal conductivity of ferrofluids prepared with different fatty acids as surfactants depends on the degree of unsaturation of the carbon chain of fatty acids with the same number of carbon atoms [18]. An important contribution of the thermal conductivity of a nanofluid is the aggregation of the NPs. It has been observed that aggregation of metallic or plasmonic NPs leads to both an enhancement of the conversion of electromagnetic energy to heat [19, 20] and of thermal conductivity [21]. Particularly, ferrofluids display a strong enhancement of the thermal conductivity due to the formation of aggregates by dipolar interaction under a magnetic field [2224 ] and without an external field at high concentrations [25]. Temperature also influences the thermal conductivity by triggering of microconvection around the NPs, and Brownian motion of the NPs but some controversies still persist [26, 27].

Besides the numerous studies employing techniques 


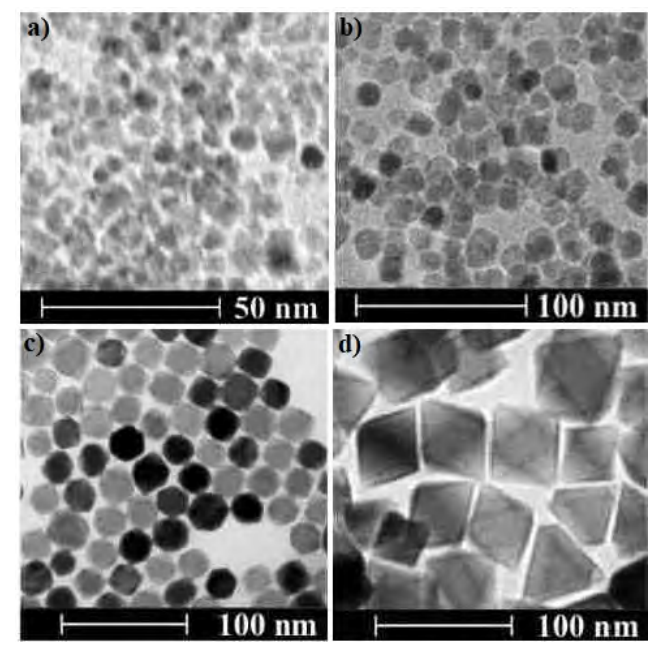

Figure 1. TEM images of the synthesized magnetite NPs: a) $6 \pm 1 \mathrm{~nm}, \mathrm{~b}) 17 \pm 1 \mathrm{~nm}$, c) $29 \pm 5 \mathrm{~nm}$, d) $63 \pm 7 \mathrm{~nm}$.

that allow direct access to the thermal conductivity and heat capacity of nanofluids, there are few studies that employ experimental techniques that allow directly obtaining thermal diffusivity. Available data show that the thermal diffusivity of the nanocolloid is higher than that of the base fluid, increasing by increasing the concentration of NPs [28, 29], and is an increasing function of the NPs size [30]. By employing the collinear mirage effect, Shibli et al. observed an increase of the thermal diffusivity of a ferrofluid as a function of concentration and $\mathrm{pH}$ [31]. In contrast to the role of magnetic field and concentration of nanoparticles on the thermal conductivity, there is a lack of data about the role of NPs size in the thermal diffusivity of nanocolloids. In this paper, we report the first study to our knowledge, on the thermal diffusivity of highly diluted colloids of superparamagnetic magnetite NPs in the zero-field and its dependence on NP size using the time-resolved mode-mismatched dualbeam thermal lens technique.

\section{MATERIALS AND METHODS}

The magnetite NPs were synthesized using thermal decomposition [32]. Figure 1 shows images by transmission electron microscopy (TEM JEM-1200EX-II, JEOL) of particles with average sizes of $6 \pm 1,17 \pm 1,29 \pm 5$ and $63 \pm 7$ $\mathrm{nm}$, where the value and error were obtained according to the protocols given in reference [33]. The phase of the NPs was determined by selected area electron diffraction (SAED). Four stable aqueous colloids were prepared by dispersing the prepared magnetite NPs with approx- imately the same number density, $10^{10} \mathrm{~mL}^{-1}$. Such high dilutions are necessary due to the strong optical absorption of the ferrofluids at the wavelength of the laser used $[34,35]$. At that concentration, the mass density of the colloids is almost that of water.

Figure 2 shows representative SAED rings of the samples and a simulation of the pattern of magnetite from reference [36], which are in good match with the d-spaces, and exhibit the high polycrystallinity of magnetite. Determinations of the x-ray diffraction pattern of the samples (Ultima IV-Rigaku) also show a matching of about $94 \%$ with that of synthetic magnetite of powder diffraction file (PDF) 000-88-0315.

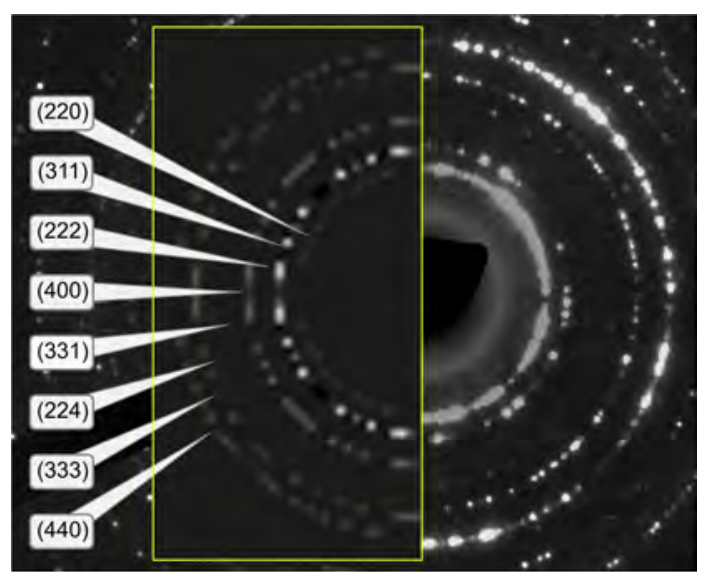

Figure 2. Characteristic SAED of the magnetite NPs of $29 \pm$ $5 \mathrm{~nm}$. The yellow squared region represents the diffraction simulation of magnetite from Ref. [36], which agrees with the experimental result.

The photothermal technique employed in this work to measure the thermal diffusivity is the time-resolved mode-mismatched dual-beam thermal lens technique, which consists in inducing a temperature gradient using a nonradiative decay processes following an optical excitation of the sample. Pulses of ms-width of a continuous wave (cw) single-mode $\mathrm{TEM}_{00}$ laser beam, obtained by a mechanical shutter, are used to excite the sample and to induce a thermal lens, which is probed by a second $\mathrm{cw}$ single-mode $\mathrm{TEM}_{00}$ laser. The mode-mismatched dualbeam thermal lens technique apparatus is the usual one described elsewhere [37].

The probe beam propagates along the $z$-direction. Photodetectors are used to monitor the temporal dependence of the transmitted probe-beam intensity in the far field at the center of the probe beam, $\Gamma(t)$, which can be written as [37, 38]:

$$
\Gamma(t)=\frac{I(t)}{I(0)}=\left\{1-\frac{\theta}{2} \tan ^{-1}\left(\frac{2 M V}{\left[(1+2 M)^{2}+V^{2}\right] \frac{t_{c}}{2 t}+1+2 M+V^{2}}\right)\right\}^{2},
$$


where

$$
\begin{aligned}
V & =\frac{Z_{1}}{Z_{c}}+\frac{Z_{c}}{Z_{2}}\left[\left(1+\frac{Z_{1}}{Z_{c}}\right)^{2}\right] \\
\theta & =-\frac{P_{e} \alpha_{o} L_{e f f}}{\kappa \lambda_{p}}\left(\frac{d n}{d T}\right) \\
M & =\left(\frac{w_{1 p}}{w_{o e}}\right)^{2}
\end{aligned}
$$

Here, $I(0)$ is the intensity measured by the photodetector at $t=0$ at the center of the probe beam, $\alpha_{o}$ is the linear optical absorption coefficient, $P_{e}$ is the excitation beam power, $\lambda_{p}$ is the probe laser wavelength, $L_{e f f}=1-e^{-\alpha_{0} L} / \alpha_{0}$ is the effective thickness of a sample of thickness $L, Z_{c}=\pi w^{2} / \lambda$ is the confocal distance, $d n / d T$ is the thermo-optic coefficient or variation of the refractive index of sample with temperature, $w_{o i}(i=p, e)$ is the minimum radius of the probe and the excitation beam, respectively, $w_{1 p}$ is the beam radius of the probe beam inside the sample, $Z_{i}(i=1,2)$ are the distances of the sample to the position of the minimum radius of the probe beam and the iris, respectively and $t_{c}$ is the characteristic thermal time constant. The effective thermal diffusivity of the medium is given by:

$$
D=\frac{w_{o e}^{2}}{4 t_{c}}
$$

Parameters $\theta$ and $t_{c}$ were obtained by fitting the normalized thermal lens signal as a function of time $(\Gamma)$ to Eq. 1. Finally the thermal diffusivity could be calculated from Eq. 5.

In our experimental setup, the excitation and probe beam had wavelengths of $532 \mathrm{~nm}$ and $632.8 \mathrm{~nm}$, with beam waists of $w_{o p}=66.5 \mu \mathrm{m}$, and $w_{o e}=38 \mu \mathrm{m}$, respectively. The geometrical parameters of the setup were $M=36.9, V=9.35, Z_{1}=6.7 \mathrm{~cm}, Z_{2}=404 \mathrm{~cm}$, and $w_{1 p}=404 \mu \mathrm{m}$. The sample was placed in a quartz cell of $0.5 \mathrm{~cm}$ of optical path length inside an oven and the temperature was fixed at $36.5 \pm 0.5{ }^{\circ} \mathrm{C}$. Figure 3 shows a schematic diagram of the laser beams inside the sample contained in the quartz cell.

Before each measurement, the sample was placed in an ultrasonic bath for $\sim 30$ min for homogenization.

\section{EXPERIMENTAL RESULTS}

Figure 4 shows typical normalized thermal lens evolution signals for the four different samples of ferrofluids. The solid lines in Figure 4 are best fit to Eq. 1, and the error bars are the standard deviation of 30 measurements.

The characteristic thermal time constant for the emergence of the induced thermal lens in the samples is in the millisecond $(\mathrm{ms})$ time scale, which is expected from a photothermal effect.

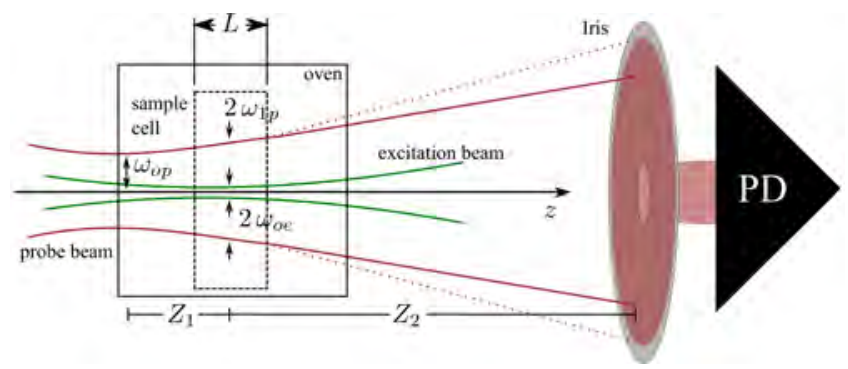

Figure 3. Representation of the positions of the laser beams in the sample for the two-beam thermal lens experimental setup. PD: photodetector, L: optical path length of the cuvette. Dotted red line represents the trajectory of the probe beam without the thermal lens effect. Solid red line represents the actual trajectory of the probe beam.

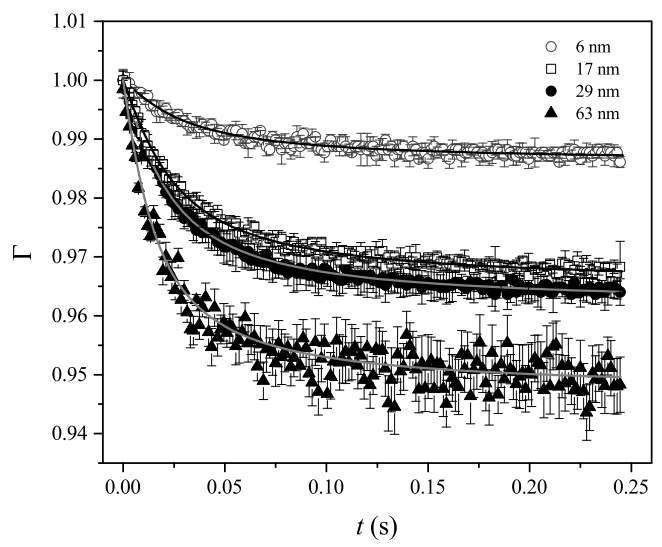

Figure 4. Typical time evolution of the normalized thermal lens signal for particles of different sizes. Solid curves are best fit to Eq. 1.

Figure 5 shows the thermal diffusion coefficient for the samples as a function of size, with all of them at the same concentration given in number density. In addition, the value of the thermal diffusivity of pure water that is obtained by this technique is shown, which agrees with the values found in the literature [39, 40]. The thermal diffusivity increases as a function of the size of the NPs, showing an enhancement of about $100 \%$ by increasing the size by a factor of 6 . It is important to note that our measurements were done on samples with the same number density. To analyze the dependence of thermal transport properties, measurements were usually done by maintaining constant concentration, given as a percen volume concentration, volume fraction or mass-to-volume ratio, for which number density is not constant. Thus, the opposite trend observed in our experiment is apparent; maintaining the number density constant, samples made of NPs of different sizes have different volume concentrations. The same data can be presented in another way. 


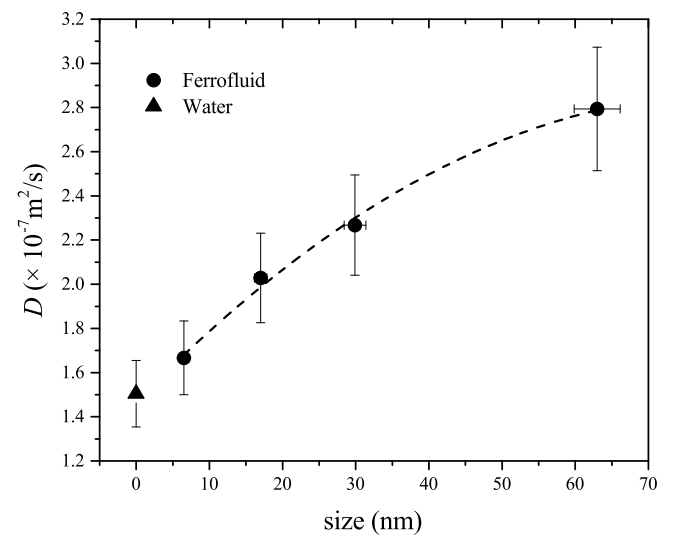

Figure 5. Thermal diffusivities of the nanofluids as function of the size of the NPs measured at $36.5^{\circ} \mathrm{C}$ on samples with the same number density of NPs. For comparison, the thermal diffusivity of water, measured at the same temperature and experimental conditions, is also shown. The dashed line is a guide for the eye.

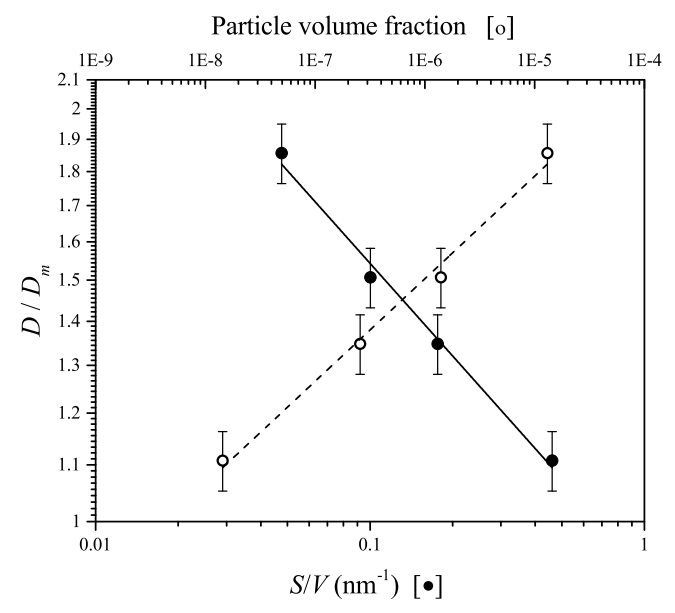

Figure 6. Thermal diffusivity of the ferrofluids normalized by that of the pure water as a function of the surface-to-volume ratio, at $36.5^{\circ} \mathrm{C}$. Solid and dashed lines are the best linear fittings.

Figure 6 shows a semi-log plots of the measured diffusion coefficient of the ferrofluids $(D)$ normalized by the value measured in pure water $\left(D_{m}\right)$. Filled dots display the value of the normalized diffusion coefficient as a function of the surface-to-volume ratio $(S / V)$ of the NPs. Data shows that the value of $D / D_{m}$ diminishes as a function of $S / V$ ( i.e., the bigger the particle, the lower the $S / V$ ratio and the bigger the relative diffusion coefficient). A linear fitting of the semi-log plot gives the angular coefficient of $-0.22 \pm 0.01$. On the other hand, the same graph shows a semi-log plot of $D / D_{m}$ as a function of the particle volume fraction of the ferrofluids. The normalized diffusion coefficient increases as a function of the particle volume fraction. Such a behavior of the normalized diffusion coefficient agrees with that observed by Shibli et al. [31], and a linear fitting gives a slope of $0.075 \pm 0.005$. The thermal diffusion coefficient is defined in terms of the thermal conductivity $\kappa$ and specific heat $c_{p}$ by $D=\frac{\kappa}{\rho c_{p}}$, where $\rho$ is the density of the nanofluid. The product of density and specific heat, $\rho c_{p}$, is the specific volumetric heat capacity of the nanofluid. The expected behavior has been observed with ferrofluids at high concentrations in the zero-field [12]. Zhou et al. [41] showed that the specific volumetric heat capacity is approximately constant as a function of the volumetric fraction. It is worth mentioning that the different shapes of the NPs could take a minor role in our results, as shown in previous works [42]. We can also to discard other factors, such as thermodiffusion or Soret effect, which occurs in a time scale by two orders higher than that employed in our experiment [43], and the existence of clusters, because of the measurements to be done at the zero-field and on samples with low concentrations. On the other hand, a possible contribution to the observed results could be the role of the surfactants (oleic acid, oleylamine and poly(vinylpyrrolidone)) in the thermal conductivity due to the quantity and proportion of surfactants employed to stabilize the nanocolloid increases with the size of the NPs.

\section{CONCLUSIONS}

In summary, we have measured the thermal diffusivity of ferrofluid made with magnetite NPs of different sizes using the thermal lens technique. The results have shown that the thermal diffusivity, for a fixed number density of NPs, is an increasing function of the NP size, showing an increase by a factor of 2 by increasing the size of the NPs by a factor of 6 . Such a behavior of the thermal diffusivity can be attributed to the increasing particle volume fraction and to the capping of the NPs.

\section{ACKNOWLEDGMENTS}

The authors acknowledge the National Institute of Science and Technology in Complex Fluids (INCT-FCx) and the Brazilian agencies $\mathrm{CNPq}$, CAPES, Fundação Araucária and Secretaria de Ciência, Tecnologia e Ensino Superior do Paraná for financial support.
[1] R. E. Rosensweig, Ferrohydrodynamics (Dover, New York 1997).
[2] K. Raj and R. Moskowitz, J Magn. Magn. Mater. 85, 233 (1990). 
[3] C. Sun, J. S. H. Lee, and M. Zhang, Adv. Drug Deliver. Rev. 60,1252 (2008).

[4] Z. Hedayatnasab, F. Abnisa, and W. M. A. W. Daud, Mater. Des. 123, 174 (2017).

[5] J. Beik, Z. Abed, F. S. Ghoreishi, S. Hosseini-Nami, S. Mehrzadi, A. Shakeri-Zadeh, and S. K. Kamrava, J Controlled Release 235, 205 (2016).

[6] S. Freddi, L. Sironi, R. D'Antuono, D. Morone, A. Dona, E. Cabrini, L. D'Alfonso, M. Collini, P. Pallavicini, G. Baldi, D. Maggioni, and G. Chirico, Nano Lett. 13, 2004 (2013).

[7] S. Bhatt, R. Kumar, and M. Kumar, Mod. Phys. Lett. B 31, 1750011 (2017).

[8] B. X. Wang, L.P.g Zhou, and X. F. Peng, Int. J Thermophys. 27, 139 (2006).

[9] I. Avramov and M. Michailov, J Phys-Condens. Mat. 20, 295224 (2008).

[10] M. C. Lu and C. H. Huang, Nanoscale Res. Lett. 8, 292 (2013).

[11] R. Hentschke, Nanoscale Res. Lett. 11, 88 (2016).

[12] N. S. S. Mousavi and S. Kumar, Int. J Therm. Sci. 84, 267 (2014).

[13] M. Higano, A. Miyagawa, K. Saigou, H. Masuda, and H. Miyashita, Int. J Thermophys. 20, 207 (1999).

[14] G. Paul, M. Chopkar, I. Manna, and P. K. Das, Renew. Sust. Energ. Rev. 14, 1913 (2010).

[15] H. Xie, W. Yu, Y. Li, and L. Chen, Nanoscale Res. Lett. 6, 124 (2011).

[16] D. H. Kumar, H. E. Patel, V. R. R. Kumar, T. Sundararajan, T. Pradeep, and S. K. Das, Phys. Rev. Lett. 93, 144301 (2004).

[17] W. Yu and S.U.S. Choi, J Nanopart. Res. 5, 167 (2003).

[18] R. Lenin, P. Alias Joy, J Colloid Interf. Sci. 506, 162 (2017).

[19] C. Yang, H. Sui, X. Li, J. Han, X. Luo, H. Zhang, H. Sun, H. Sun, Y. Zhouc, and B. Yang, CrystEngComm 15, 3490 (2013).

[20] H. H. Richardson, Z. N. Hickman, A. O. Govorov, A. C. Thomas, W. Zhang, and M. E. Kordesch, Nano Lett. 6, 783 (2006).

[21] V. M. Lenart, R. F. Turchiello, G. F. Goya, and S. L. Gómez, Braz. J Phys. 45, 213 (2015).

[22] J. Philip, P. D. Shima, and B. Raj, Appl. Phys. Lett. 91, 203108 (2007).
[23] M. Bahiraei and M. Hangi, J Magn. Magn. Mater. 374, 125 (2015).

[24] C. L. Altan, A. Elkatmis, M. Yüksel, N. Aslan, and S. Bucak, J Appl. Phys. 110, 093917 (2011).

[25] H. Zhua, C. Zhang, S. Liu, Y. Tang, and Y. Yin, Appl. Phys. Lett. 89, 023123 (2006).

[26] Y. Xuan, Q. Li, and W. Hu, AIChE J 49, 1038 (2003).

[27] P. Keblinski, R. Prasher, and J. Eapen, J Nanopart. Res. 10,1089 (2008).

[28] X. Zhang, H. Gu, and M. Fujii, J. Appl. Phys. 100, 044325 (2006).

[29] S. M. S. Murshed, K. C. Leong, and C. Yang, J Phys. D: Appl. Phys. 39 (2006) 5316.

[30] E. Shahriari, W. M. Mat Yunus, and R. Zamiri, J. Europ. Opt. Soc. Rap. Public. 8, 13026 (2013).

[31] S. M. Shibli, A. L. L. Dantas, and A. Bee, and , Braz. J Phys. 31, 418 (2001).

[32] V. M. Lenart, S. L. Gómez, M. P. Calatayud, and G. R. F. Goya, arXiv:1402.1134 (2014).

[33] A. Jillavenkatesa, S. J. Dapkunas, and L. S. H. Lum, "Nist recommended practice guide: particle size characterization", Special Publication (NIST SP)-960-1 (2001).

[34] D. Espinosa, D. Soga, S. Alves, L. de Boni, S. C. Zílio, and A. M. Figueiredo Neto, J Opt. Soc. Am. B 29, 280 (2012).

[35] M. E. Sadat, M. K. Baghbador, A. W. Dunn, H. P. Wagner, R. C. Ewing, J. Zhang, H. Xu, G. M. Pauletti, D. B. Mast, and D. Shi, Appl. Phys. Lett. 105, 091903 (2014).

[36] W. H. Bragg, Nature 95, 561 (1915).

[37] M. L. Baesso, J. R. D. Pereira, A. C. Bento, A. J. Palangana, A. M. Mansanares, and L. R. Evangelista, Braz. J. Phys. 28, 359 (1998).

[38] J. Shen, R. D. Lowe, and R. D. Snook, Chem. Phys. 165, 385 (1992).

[39] D. W. James, J Mater. Sci. 3, 540 (1968).

[40] P. R. B. Pedreira, L. Hirsch, J. R. D. Pereira, A. N. Medina, A. C. Bento, and M. L. Baesso, Rev. Sci. Instrum. 74, 808 (2003).

[41] L. P. Zhou, B. X. Wang, X. F. Peng, X. Z. Du, and Y. P. Yang, Adv. Mech. Eng. 172085 (2010).

[42] S. Ferrouillat, A. Bontemps, O. Poncelet, O. Soriano, J. A. Grus, Appl. Therm. Eng. 51, 839 (2013).

[43] S. I. Alves, A. Bourdon, and A. M. Figueiredo Neto, J Magn. Magn. Mater. 289, 285 (2005). 